\title{
Low-density lipoprotein cholesterol within the normal range and nonalcoholic fatty liver disease in the non-obese Chinese population: a secondary analysis based on a cross-sectional study
}

\section{Zhigang (N/A) Xu}

Capital Medical University https://orcid.org/0000-0001-5404-0743

Baohong Xu ( $\nabla$ bhxu22@126.com)

https://orcid.org/0000-0001-7834-889X

\section{Research}

Keywords: LDL-c, NAFLD, non-obese Chinese, non-linear

Posted Date: April 28th, 2020

DOI: https://doi.org/10.21203/rs.3.rs-22525/v1

License: (a) (1) This work is licensed under a Creative Commons Attribution 4.0 International License. Read Full License 


\section{Abstract \\ Background}

Evidence regarding the relationship between normal low-density lipoprotein cholesterol (LDL-C) levels and non-alcoholic fatty liver disease (NAFLD) was limited. Therefore, this dissertation seeks to investigate the relationship between LDL-c and NAFLD in the non-obese Chinese population after adjusting for other covariates.

\section{Methods}

The present study was a cross-sectional study. A total of 183903 non-obese participants were involved in a Wenzhou Medical Center of Wenzhou People's Hospital from 2010 to 2014. The target independent variable and the dependent variable were LDL-c measured at baseline and NAFLD respectively. Covariates involved in this study included SEX, AGE, BMI, SBP, DBP, FPG, ALB, ALT, AST, BUN, Cr, TG, TC, HDL-C, UA. It was noted that the entire study was completed by Dan-Qin Sun et al., and uploaded the data to the DATADRYAD website. The author only used this data for secondary analysis.

\section{Results}

The average age of 183903 selected participants was $41.0 \pm 14.1$ years old,and about $49.6 \%$ of them was male. After adjusting potential confounders (SEX, AGE, BMI, FPG, ALB, GLB, ALT, AST, GGT, BUN, Cr, TG, TC, HDL-C, UA), non-linear relationship was detected between normal LDL-c levels and NAFLD, whose point was 1.51. The effect sizes and the confidence intervals on the left and right sides of inflection point were $0.87(0.64,1.18)$ and $1.79(1.67,1.92)$, respectively.

\section{Conclusion}

The relationship between normal LDL-c levels and NAFLD is non-linear. Normal LDL-c levels was positively correlated with NAFLD when LDL-c was more than 1.51 .

\section{Introduction}

Non-alcoholic fatty liver disease (NAFLD) results from hepatic fat accumulation in the absence of quantities of alcohol and any secondary cause ${ }^{[1-5]}$. Histologically, It comprises a range of pathologic conditions including simple nonalcoholic steatosis, nonalcoholic steatohepatitis (NASH) and hepati cirrhosis $^{[6,7]}$. NAFLD is a major, worldwide public health problem and its incidence among adults and adolescents has increased rapidly in recent years. Salvoza eta ${ }^{[8]}{ }_{\text {found }}$ that the prevalence of NAFLD is $30-40 \%$ in the United States, $2-44 \%$ in Europe and $15-45 \%$ in Asia, respectively. 
One meta-analysis reports that it is the relatively high prevalence of NAFLD found in the Asian population $(27 \%)^{[9-11]}$. The prevalence rate of NAFLD in the general population of China varies from $24.77-43.91 \%$ in recent years ${ }^{[11,12]}$.

NAFLD is associated with an increased risk of developing to severe and decompensated liver diseases such as cirrhosis and hepatocellular carcinoma ${ }^{[13-15]}$. In addition, NAFLD also can increase the risk of cardiovascular disease, type 2 diabetes and chronic kidney disease ${ }^{[16-19]}$.

Obesity is a well-known risk factor for the development of NAFLD, and NAFLD is now considered to be one of the most prevalent manifestations of obesity-related metabolic syndrome (MS) ${ }^{[20]}$. However, there is growing evidence that the prevalence of NAFLD in non-obese individuals is not uncommon. Especially in Asia, where people are less obese than in Western countries and the prevalence of NAFLD is increasing with time ${ }^{[21]}$. Studies have shown that approximately $15-21 \%$ of the Asian patients with NAFLD are nonobese.

Dyslipidemia is a comorbidity of NAFLD, which can lead to hypertriglyceridaemia, reductions in highdensity lipoprotein cholesterol (HDL-c), an increase in the size of very low-density lipoprotein and lowdensity lipoprotein cholesterol (LDL-c) ${ }^{[22]}$. In addition, more and more studies have shown that LDL-c is involved in the development of NAFLD and non-alcoholic steatohepatitis (NASH) ${ }^{[23-25]}$. This mechanism is still unclear, but insulin resistance and disorders of fat and sugar metabolism may explain some reasons $^{[26-28]}$. In addition, damage to the LRP6 receptor is also responsible ${ }^{[29,30]}$.

In any case, so far, no studies have explored the curves relationship between LDL-c and NAFLD. In biomedical research, connection between exposures and outcomes may be non-linear. In that case, researchers need a more effective method to deal with non-linear relationship. In this study, we use curves to fit LDL-c and NAFLD.

\section{Methods}

\section{Data source}

We obtained data from 'DATADRYAD' database (www.Datadryad.org). This website permitted users to freely download the raw data. According to Drya Terms of Service, we cited Dryad data package in the present study. (Sun, Dan-Qin; Wu, Sheng-Jie; Liu, Wen-Yue; Wang, Li-Ren; Chen, Yi-Ran; Zhang, Dong-Chu; et al. (2016): Data from: Association of low-density lipoprotein cholesterol within the normal range and NAFLD in the non-obese Chinese population: a cross-sectional and longitudinal study. Dryad. Collection. https://doi.org/10.5061/dryad.1n6c4). Variables included in the database file were as follows: age, sex, body mass index (BMI), fasting plasma glucose(FPG), albumin (ALB), globulin (GLB), alanine aminotransferase (ALT), aspartate transaminase (AST), Y-glutamyltranspeptidase (GGT), blood urea nitrogen (BUN), creatinine (CR), triglyceride (TG), total cholesterol (TC), high-density lipoprotein 
cholesterol (HDL-c), uric acid (UA), total bilirubin (TBIL), low density lipoprotein (LDL-c), non-alcoholic fatty liver disease (NAFLD).

\section{Study population}

Sun D-Q, Wu S-J, Liu W-Y, et al[ ${ }^{[31]}$ completed the entire study. In order to allow to understand the entire research process more clearly, we have outlined the steps of the study here. The specific details are described in the original Reported by Sun D-Q, Wu S-J, Liu W-Y, et al. They conducted a cross-sectional study at Wenzhou Medical Center of Wenzhou People's Hospital, zhejiang city, china from 2010 to 2014. Participants received a medical health examination including the biochemical measurements and abdominal ultrasonography. A total of 183903 non-obese individuals were enrolled and selected according to exclusion standards. Exclusion standards: (1) Individuals were reported excess alcohol consumption (>140 g/week for men and > $70 \mathrm{~g} /$ week for women), (2) those taking antihypertensive agents, antidiabetic agents, lipid-lowing agents, (3) other known causes of chronic liver disease, (4) LDL-c of $>3.12 \mathrm{mmol} / \mathrm{L}$, (5) BMl of $\geq 25 \mathrm{~kg} / \mathrm{m}^{2}$, (6) Missing data. Researchers obtained information (values) of NAFLD, LDL-C and other covariants at baseline. This study was performed by Chinese researcher Sun D-Q, Wu S-J,Liu W-Y,et al. at Wenzhou Medical Center of Wenzhou People's Hospital, zhejiang city, china. In the previously published article ${ }^{[31,32]}$, Sun D-Q, Wu S-J,Liu W-Y, et al. has clearly stated that: Verbal informed consent was obtained from each participant before their participation in the study. The personal information of participants was erased and replaced by the health examination number. The research protocol of the study was approved by the ethics committee of Wenzhou People's Hospital. Informed consent was obtained from all Participants.

\section{Measurement of LDL-C, NAFLD and other covariants}

The biochemical measurements included albumin (ALB), alanine aminotransferase (ALT), aspartate aminotransferase (AST), fasting plasma glucose (FPG), globulin (GLB), y-glutamyltranspeptidase (GGT), blood urea nitrogen (BUN), creatinine (Cr), uric acid (UA), total bilirubin (TBIL), total cholesterol (TC), triglyceride (TG), high-density lipoprotein cholesterol (HDL-c) and low-density lipoprotein cholesterol (LDLc). All values were measured by an automated analyser (Abbott AxSYM) using standard methods. Medical history and a health habit inventory were taken by a physician. BMI $\left(\mathrm{kg} / \mathrm{m}^{2}\right)$, used as an index of body fat, was calculated as weight in kilograms divided by height in $\mathrm{m}^{2}$. The ultrasound criteria for the diagnosis of fatty liver were based on those suggested by the Chinese Liver Disease Association. The assessment of NAFLD described in detail in the original.

\section{Statistical analysis}

Continuous variables were expressed as mean \pm standard deviation (normal distribution) or median (quartile) (skewed distribution), and categorical variables were expressed in frequency or as a percentage. The One-Way Anova (normal istribution), Kruscal Whallis $\mathrm{H}$ (skewed distribution) test and chi-square tests (categorical variables) were used to determine any statistical differences between the means and proportions of the groups. Univariate linear regression model was used to evaluate the associations between LDL-c and NAFLD. Both non-adjusted and multivariate adjusted models were listed in the paper. 
According to the recommendation of STROBE statement, we simultaneously showed the results of unadjusted, minimally adjusted analyses and those from fully adjusted analyses. Whether the covariances were adjusted determined by the following principle: when added to this model, changed the matched odds ratio by at least $10 \%{ }^{[33]}$. Besides, we also used generalized additive model (GAM) to identify the non-linear relationship. If the non-linear correlation was observed, a two-piecewise linear regression model was performed to calculate the threshold effect of the LDL-C and NAFLD in terms of the smoothing plot. When the ratio between LDL-c and NAFLD appears obvious in smoothed curve, recursive method calculates automatically the inflection point, where the maximum model likelihood will be used $^{[34]}$. All of the analyses were performed with the statistical software packages R (http://www. Rproject.org, The R Foundation) and EmpowerStats (http://www.empowerstats.com, X\&Y Solutions, Inc., Boston, MA). $P$ values less than 0.05 (two-sided) were considered statistically significant.

\section{Results}

\section{Baseline characteristics of selected participants}

A total of 183903 participants were sclected for the final data analysis based on the inclusion and exclusion criteria. We showed baseline characteristics of these selected participants in Table 1 according to Quartile of LDL-c. In general, the average age of the 183903 selected participants was $41.0 \pm 14.1$ years old, and about $49.6 \%$ of them were male. statistically significant differences were detected in AGE, BMI, HDL, ALT, AST, GGT, BUN, Cr, TG, FPG, TC, UA, GLB and ALB, among different LDL-c groups (all p values < 0.01). Participants wih the highest group of LDL-C (Q4) had the higher values in AGE, BMI, ALT, AST, GGT, BUN, CR, TG, FPG, TC, UA, GLB and ALB than those of the other groups. The opposite pattern were observed in HDL-C. 
Table 1

Baseline Characteristics of participants $(\mathrm{N}=183903)$

\begin{tabular}{|c|c|c|c|c|c|}
\hline LDL-C & $\begin{array}{l}\text { Q1 (0.41- } \\
1.91)\end{array}$ & $\begin{array}{l}\text { Q2 (1.92- } \\
2.26)\end{array}$ & $\begin{array}{l}\text { Q3 (2.27- } \\
2.60)\end{array}$ & $\begin{array}{l}\text { Q4 (2.61- } \\
3.12)\end{array}$ & $\begin{array}{l}\mathrm{P}- \\
\text { value }\end{array}$ \\
\hline Number & 45352 & 45917 & 45679 & 46952 & \\
\hline Age, years & $38.0 \pm 13.9$ & $39.3 \pm 13.4$ & $41.7 \pm 13.7$ & $44.8 \pm 14.2$ & $\begin{array}{l}< \\
0.001\end{array}$ \\
\hline $\mathrm{BMl}, \mathrm{kg} / \mathrm{m}^{2}$ & $20.8 \pm 2.2$ & $21.2 \pm 2.1$ & $21.7 \pm 2.1$ & $22.1 \pm 1.9$ & $\begin{array}{l}<.001 \\
0.001\end{array}$ \\
\hline $\begin{array}{l}\text { FPG log2 } \\
\text { transform }\end{array}$ & $2.3 \pm 0.2$ & $2.3 \pm 0.2$ & $2.4 \pm 0.2$ & $2.4 \pm 0.2$ & $\begin{array}{l}< \\
0.001\end{array}$ \\
\hline ALB, $g / L$ & $44.4 \pm 2.9$ & $44.6 \pm 2.8$ & $44.7 \pm 2.8$ & $44.7 \pm 2.8$ & $\begin{array}{l}<.001 \\
0.00\end{array}$ \\
\hline GLB, g/L & $29.3 \pm 4.0$ & $29.3 \pm 3.8$ & $29.3 \pm 3.8$ & $29.5 \pm 3.8$ & $\begin{array}{l}<.001 \\
0.001\end{array}$ \\
\hline $\begin{array}{l}\text { ALT log2 } \\
\text { transform }\end{array}$ & $4.0 \pm 0.7$ & $4.1 \pm 0.7$ & $4.2 \pm 0.8$ & $4.3 \pm 0.8$ & $\begin{array}{l}< \\
0.001\end{array}$ \\
\hline $\begin{array}{l}\text { AST log2 } \\
\text { transform }\end{array}$ & $4.4 \pm 0.4$ & $4.4 \pm 0.4$ & $4.4 \pm 0.4$ & $4.5 \pm 0.4$ & $\begin{array}{l}<.001 \\
0.00\end{array}$ \\
\hline $\begin{array}{l}\text { GGT log2 } \\
\text { transform }\end{array}$ & $4.3 \pm 0.8$ & $4.5 \pm 0.8$ & $4.6 \pm 0.9$ & $4.7 \pm 0.9$ & $\begin{array}{l}< \\
0.001\end{array}$ \\
\hline $\begin{array}{l}\text { BUN log2 } \\
\text { transform }\end{array}$ & $2.0 \pm 0.4$ & $2.1 \pm 0.4$ & $2.1 \pm 0.4$ & $2.1 \pm 0.4$ & $\begin{array}{l}<.001 \\
0.001\end{array}$ \\
\hline Cr log2 transform & $6.2 \pm 0.3$ & $6.2 \pm 0.3$ & $6.3 \pm 0.3$ & $6.3 \pm 0.3$ & $\begin{array}{l}<.001 \\
0.001\end{array}$ \\
\hline TG log2 transform & $-0.1 \pm 0.8$ & $0.1 \pm 0.7$ & $0.3 \pm 0.7$ & $0.4 \pm 0.7$ & $\begin{array}{l}< \\
0.001\end{array}$ \\
\hline $\mathrm{TC}, \mathrm{mmol} / \mathrm{L}$ & $3.8 \pm 0.5$ & $4.3 \pm 0.5$ & $4.8 \pm 0.5$ & $5.3 \pm 0.5$ & $\begin{array}{l}<.001 \\
0.001\end{array}$ \\
\hline $\mathrm{HDL}-\mathrm{c}, \mathrm{mmol} / \mathrm{L}$ & $1.5 \pm 0.4$ & $1.4 \pm 0.4$ & $1.4 \pm 0.3$ & $1.4 \pm 0.3$ & $\begin{array}{l}<.001 \\
0.001\end{array}$ \\
\hline $\mathrm{UA}, \mu \mathrm{mol} / \mathrm{L}$ & $264.5 \pm 85.9$ & $277.1 \pm 87.6$ & $289.5 \pm 88.7$ & $300.9 \pm 89.1$ & $\begin{array}{l}<.001 \\
0.00\end{array}$ \\
\hline TBIL, $\mu \mathrm{mol} / \mathrm{L}$ & $12.4 \pm 5.4$ & $12.3 \pm 5.0$ & $12.3 \pm 5.0$ & $12.2 \pm 4.8$ & 0.815 \\
\hline
\end{tabular}

ALB, albumin; GLB, globulin, GGT, y-glutamyltranspeptidase, ALT, alanine aminotransferase; AST, aspartate aminotransferase; BMI, body mass index; BUN, blood urea nitrogen; $\mathrm{Cr}$, creatinine; FPG, fasting plasma glucose; HDL-c, high-density lipoprotein cholesterol; LDL-c, low-density lipoprotein cholesterol; NAFLD, non-alcoholic fatty liver disease; TC, total cholesterol; TG, triglyceride; UA, uric acid; TBIL, total bilirubin. 


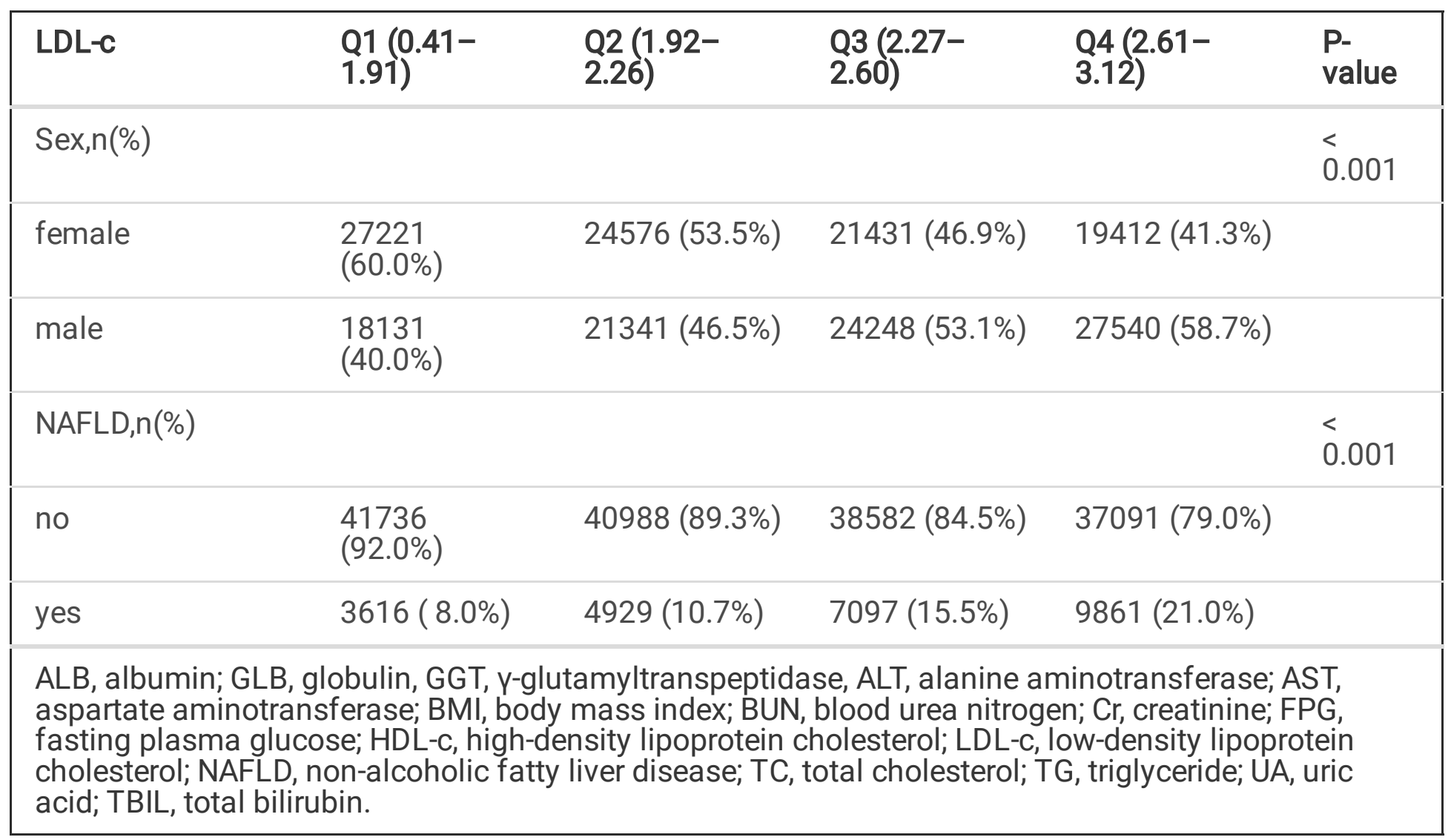

\section{Univariate analysis}

We listed the results of univariate analyses in Table 2. The results of univariate analysis showed that AGE, BMI, ALT, AST, GGT, BUN, CR, TG, FPG, TC, UA, TBIL, GLB and ALB were correlated with NAFLD. We found that AGE $1.03(1.03,1.03)$, BMI $2.08(2.06,2.11)$, FPG log2 transform $2.53(2.48,2.59)$, ALB 1.07 (1.06, 1.07), GLB 1.01 (1.01, 1.01), ALT log2 transform 3.05 (2.99, 3.11), AST log2 transform 3.18 (3.09, 3.28), GGT log2 transform $2.72(2.68,2.77)$, BUN log2 transform $1.87(1.81,1.93)$, Cr log2 transform 3.33 $(3.20,3.47)$, TG $\log 2$ transform $4.67(4.57,4.76)$, TC $1.74(1.71,1.77)$, and UA $1.01(1.01,1.01)$ are risk factors for NAFLD, however HDL-c $0.11(0.10,0.11)$ is a protective factor for NAFLD. 
Table 2

The results of univariate analysis

\begin{tabular}{|c|c|c|c|}
\hline Covariate & Statistics & OR $(95 \% \mathrm{Cl})$ & $P$-value \\
\hline Age, years & $40.98 \pm 14.06$ & $1.03(1.03,1.03)$ & $<0.001$ \\
\hline $\mathrm{BMI}, \mathrm{kg} / \mathrm{m}^{2}$ & $21.43 \pm 2.13$ & $2.08(2.06,2.11)$ & $<0.001$ \\
\hline FPG log2 transform & $5.08 \pm 0.53$ & $2.53(2.48,2.59)$ & $<0.001$ \\
\hline ALB, U/L & $44.59 \pm 2.84$ & $1.07(1.06,1.07)$ & $<0.001$ \\
\hline GLB, U/L & $29.34 \pm 3.86$ & $1.01(1.01,1.01)$ & $<0.001$ \\
\hline ALT log2 transform & $4.12 \pm 0.76$ & $3.05(2.99,3.11)$ & $<0.001$ \\
\hline AST log2 transform & $4.43 \pm 0.42$ & $3.18(3.09,3.28)$ & $<0.001$ \\
\hline GGT log2 transform & $4.54 \pm 0.86$ & $2.72(2.68,2.77)$ & $<0.001$ \\
\hline BUN log2 transform & $2.08 \pm 0.40$ & $1.87(1.81,1.93)$ & $<0.001$ \\
\hline Cr log2 transform & $6.26 \pm 0.33$ & $3.33(3.20,3.47)$ & $<0.001$ \\
\hline TG log2 transform & $0.19 \pm 0.76$ & $4.67(4.57,4.76)$ & $<0.001$ \\
\hline TC, $\mathrm{mmol} / \mathrm{L}$ & $4.54 \pm 0.74$ & $1.74(1.71,1.77)$ & $<0.001$ \\
\hline $\mathrm{HDL}-\mathrm{c}, \mathrm{mmol} / \mathrm{L}$ & $1.45 \pm 0.36$ & $0.11(0.10,0.11)$ & $<0.001$ \\
\hline $\mathrm{UA}, \mu \mathrm{mol} / \mathrm{L}$ & $283.13 \pm 88.90$ & $1.01(1.01,1.01)$ & $<0.001$ \\
\hline TBIL, $\mu \mathrm{mol} / \mathrm{L}$ & $12.30 \pm 5.04$ & $1.01(1.01,1.01)$ & $<0.001$ \\
\hline LDL-c, mmol/L & $2.25 \pm 0.47$ & $2.54(2.46,2.61)$ & $<0.001$ \\
\hline LDL-c (Quartile) & & & $<0.001$ \\
\hline Q1 & $45352(24.66 \%)$ & 1.0 & \\
\hline Q2 & 45917 (24.97\%) & $1.39(1.33,1.45)$ & $<0.001$ \\
\hline Q3 & 45679 (24.84\%) & $2.12(2.04,2.22)$ & $<0.001$ \\
\hline Q4 & $46952(25.53 \%)$ & $3.07(2.95,3.20)$ & $<0.001$ \\
\hline Sex,n(\%) & & & $<0.001$ \\
\hline female & $92643(50.38 \%)$ & 1.0 & \\
\hline male & 91260 (49.62\%) & $4.22(4.09,4.35)$ & \\
\hline
\end{tabular}

\section{The results of relationship between LDL-c and NAFLD}

In this study, We used Multivariate linear regression model to evaluate the associations between LDL-C and NAFLD. Meanwhile,we showed the non-adjusted and adjusted modles in Table 3. In crude model, 
LDL-c showed positive correlation with NAFLD (OR $=2.54,95 \%$ confidence interval $(\mathrm{Cl}): 2.46$ to $2.61, \mathrm{P}<$ 0.001). In minimally adjusted model (adjusted age, sex), the result have obvious changes (OR $=2.01,95 \%$ confidence interval $(\mathrm{Cl}): 1.94$ to 2.07, $\mathrm{P}<0.001)$. However,we detect the Independent effect LDL-c on NAFLD in fully adjusted model $(O R=1.70,95 \%$ confidence interval $(\mathrm{Cl}): 1.59$ to $1.82, \mathrm{P}<0.001)$. For the purpose of sensitivity analysis, we also handled LDL-c as Categorical variable (Quartile), and found that the same trend was observed as well ( $p$ for trend was less than 0.001). Besides,we also found the trend of the effect size in different model was equidistant.

Table 3

Relationship between LDL-C and NAFLD in different models

\begin{tabular}{|llll|}
\hline Exposure & Non-adjusted & Adjust I & Adjust II \\
\hline LDL-C & $2.54(2.46,2.61)<0.001$ & $2.01(1.94,2.07)<0.001$ & $1.70(1.59,1.82)<0.001$ \\
\hline LDL-c (quartile) & & & \multicolumn{1}{l}{ Ref } \\
\hline Q1 & Ref & Ref & R \\
Q2 & $1.39(1.33,1.45)<0.001$ & $1.28(1.23,1.35)<0.001$ & $1.18(1.10,1.25)<0.001$ \\
Q3 & $2.12(2.04,2.22)<0.001$ & $1.78(1.70,1.86)<0.001$ & $1.47(1.37,1.58)<0.001$ \\
Q4 & $3.07(2.95,3.20)<0.001$ & $2.33(2.23,2.43)<0.001$ & $1.76(1.62,1.91)<0.001$ \\
\hline Pfor trend & $<0.001$ & $<0.001$ & $<0.001$
\end{tabular}

Crude model: we did not adjust other covariants

Minimally adjusted Model : we adjusted age and sex

Fully adjusted Model : we adjusted AGE, SEX, BMI, ALT, AST, GGT, BUN, Cr, TG, FPG, TC, UA, HDL-C, GLB and ALB. $\mathrm{Cl}$, confidence interval; OR, odds ratio

\section{The analyses of non-linear relationship}

Because LDL-c was continuous variable, the analyses of non-linear relationship are necessary. In the present study (Fig. 1), we found that the relationship between LDL-C and NAFLD was non-linear (after adjusting AGE, SEX, BMI, ALT, AST, GGT, BUN, Cr, TG, HDL-C, FPG, TC, UA, GLB and ALB). By two-piecewise linear regression model, we calculated that the inflection point was 1.51. On the left side of the inflection point, the effect size, $95 \% \mathrm{Cl}$ and $\mathrm{P}$ value were $0.87,0.64$ to 1.18 and 0.3760 , respectively. However, On the right side of the inflection point, the effect size, $95 \% \mathrm{Cl}$ and $\mathrm{P}$ value were $1.79,1.67$ to 1.92 and $\mathrm{p}<0.0001$, respectively (Table 4).

Table 4 The results of two-piecewise linear regression model

\begin{tabular}{|l|l|l|l|}
\hline Inflection point of LDL-c & OR & $95 \%$ CI & P value \\
\hline$<1.51$ & 0.87 & $(0.64,1.18)$ & 0.3760 \\
\hline$\geq 1.51$ & 1.79 & $(1.67,1.92)$ & $<0.0001$ \\
\hline
\end{tabular}


Effect: NAFLD Cause: LDL-c Adjusted: SEX; AGE; BMI; ALB; GLB; ALT; AST; GGT; BUN; Cr; FPG; HDL-c; TG; TC; UA.

\section{Discussion}

Previous studies showed that LDL-c was associated with NAFLD, but the relationship between the risk factor LDL-C and NAFLD was not fully described. We conducted a PubMed search and three scientific papers were retrieved from the database as of the end of March 2020. All of these studies showed that LDL-c was associated with NAFLD ${ }^{[24,31,32]}$. Sun D-Q, Wu S-J,Liu W-Y, et al. found that LDL-c was associated with NAFLD in the non-obese Chinese population in a cross-sectional and longitudinal study ${ }^{[31,32]}$. In their studies, the multivariable logistic regression models were used to calculate the OR of LDL-C on NAFLD. After adjusting potential confounders (SEX, AGE, BMI, DBP, SBP, ALB, ALT, AST, BUN, Cr, FPG, HDL-C, TC, TG and UA), the OR gradually increased in Q1 to Q4 of LDL-c quartile, and the P for trend was less than 0.05 . This suggests that the connection between LDL-C and NAFLD is non-linear. However, none of them discussed the non-linear connection between LDL-c and NAFLD. To our knowledge, this is the first study to investigate the non-linear relationship between LDL-c and NAFLD.

In the present study, we used GLM and GAM models to elucidate the relationship between LDL-C and NAFLD among participants. As is shown in the fully adjusted model, LDL-c was associated with NAFLD. When we handled LDL-C as a categorical variable, the same trend was observed. However, the results obtained from GAM and two-piecewise linear regression model showed that the relationship between LDL-C and NAFLD was non-linear, and the correlations between LDL-C and NAFLD were different on the left and right sides of the inflection point $(L D L-C=1.51)$. $L D L-C$, as assessed at baseline, was not statistically significant on the left side of the inflection point, but LDL-c was positively associated with NAFLD on the right of the inflection point.

\section{Our study has a number of strengths}

(1) we not only use the generalized linear model to evaluate the linear relationship between LDL-C and NAFLD, but also use the generalized additive model to clarify the nonlinear relationship. GAM has obvious advantages in dealing with non-linear relations and it can handle the non-parametric smoothing and will fit a regression spline to the data. The use of GAM will help us to better discover the real relationships between exposure and outcome. we detect this nonlinear relationship in our study after adjusting confounding factors which were not founded by previous study. (2) this study is an observational study including unavoidable potential confounding, so we used strict statistical adjustment to minimize residual confounding.

\section{There are some limitations in our study}

(1) this study is a analytical cross-sectional study and therefore only provides weak evidence between exposure and outcome, and it is difficult to distinguish the cause and effect. (2) because the study population contains only Chinese, it may be not generalisable to other biographic ethic groups. (3) the 
study is lack of anthropometric parameters regarding central obesity, lifestyle and dietary factors. In addition, we cannot determine the severity of NAFLD diagnosed by ultrasonographic. Due to the limitation of the original data, we cannot observe the correlation between insulin resistance and NAFLD, although insulin resistance may be closely associated with NAFLD in non-obese individuals ${ }^{[26]}$.

\section{Conclusion}

Increased normal LDL-c levels are independently associated with an elevated risk of NAFLD in non-obese individuals. The relationship between normal LDL-c levels and the risk of NAFLD is non-linear. LDL-c is positively correlated with NAFLD when normal LDL-c levels is larger than $1.51 \mathrm{mmol} / \mathrm{L}$.

\section{Declarations}

\section{Acknowledgments}

We appreciate Sun D-Q, Wu S-J, Liu W-Y et al. for their spirit of scientific sharing. We also thank Changzhong Chen, Xin-Lin Chen and Chi-Chen of Yi-er college.

\section{Authors' contributions}

XZG contributed to the drafting of the manuscript. LMY and ZJ analysed and interpreted the data. XBH contributed to the conception and critical revision of the manuscript, analysis and interpretation of the data and approved the final version of the submitted manuscript. All authors read and approved the final manuscript.

\section{Funding}

No funding was received.

\section{Availability of data and materials}

The datasets analyzed during the current study are available from the corresponding author on reasonable request.

\section{Ethics approval and consent to participate}

The research ethics were not required anymore, which had been authorized in the former study according to public policy statements of the dataset.

\section{Consent for publication}

Not applicable.

\section{Competing interests}


The authors declare that they have no conflict of interest.

\section{Author details}

1Department of Gastroenterology, LuHe Hospital of Capital Medical University, 82\# Xinhuanan Road, Beijing 101149, China.

\section{References}

1. Siddiqi Z, Karoli R, Fatima J, et al. Soft Drinks Consumption and the Risk of Nonalcoholic Fatty Liver Disease[J]. J Assoc Physicians India. 2017;65:28-32.

2. Adams LA, Anstee QM, Tilg H, et al. Non-alcoholic fatty liver disease and its relationship with cardiovascular disease and other extrahepatic diseases[J]. Gut. 2017;66:1138-53. https://doi.org/10.1136/gutjnl-2017-313884.

3. Ballestri S, Zona S, Targher G, et al. Nonalcoholic fatty liver disease is associated with an almost twofold increased risk of incident type 2 diabetes and metabolic syndrome. Evidence from a systematic review and meta-analysis[J]. J Gastroenterol Hepatol, 2016,31:936-44. https://doi.org/ 10.1111/jgh.13264.

4. Lonardo A, Ballestri S, Marchesini G, et al. Nonalcoholic fatty liver disease: a precursor of the metabolic syndrome[J]. Dig Liver Dis, 2015,47:181-90. https://doi.org/ 10.1016/j.dld.2014.09.020.

5. Darmawan G, Hamijoyo L, Hasan I. Association between Serum Uric Acid and Non-Alcoholic Fatty Liver Disease: A Meta-Analysis[J]. Acta Med Indones. 2017;49:136-47.

6. Pathophysiology of Nonalcoholic Fatty Liver Disease/Nonalcoholic Steatohepatitis[J] https:

Manne V, Handa P, Kowdley KV. Pathophysiology of Nonalcoholic Fatty Liver Disease/Nonalcoholic Steatohepatitis[J]. Clin Liver Dis, 2018,22:23-37. https://doi.org/ https://doi.org/10.1016/j.cld.2017.08.007.

7. Chalasani N, Younossi Z, Lavine JE, et al. The diagnosis and management of nonalcoholic fatty liver disease: Practice guidance from the American Association for the Study of Liver Diseases[J]. Hepatology, 2018,67:328-57. https://doi.org/ 10.1002/hep.29367.

8. Salvoza NC, Klinzing DC, Gopez-Cervantes J, et al. Association of Circulating Serum miR-34a and miR-122 with Dyslipidemia among Patients with Non-Alcoholic Fatty Liver Disease[J]. PLoS One, 2016,11:e153497. https://doi.org/ 10.1371/journal.pone.0153497.

9. Wu SJ, Zou H, Zhu GQ, et al. Increased levels of systolic blood pressure within the normal range are associated with significantly elevated risks of nonalcoholic fatty liver disease[J]. Med (Baltim), 2015,94:e842. https://doi.org/10.1097/MD.0000000000000842.

10. Angulo P. Nonalcoholic fatty liver disease[J]. N Engl J Med, 2002,346:1221-31. https://doi.org/ 10.1056/NEJMra011775. 
11. Younossi ZM, Koenig AB, Abdelatif $D$, et al. Global epidemiology of nonalcoholic fatty liver diseaseMeta-analytic assessment of prevalence, incidence, and outcomes[J]. Hepatology, 2016,64:73-84. https://doi.org/ 10.1002/hep.28431.

12. Sung KC, Kim SH. Interrelationship between fatty liver and insulin resistance in the development of type 2 diabetes[J]. J Clin Endocrinol Metab, 2011,96:1093-7. https://doi.org/ 10.1210/jc.20102190.

13. Amarapurkar DN, Hashimoto E, Lesmana LA, et al. How common is non-alcoholic fatty liver disease in the Asia-Pacific region and are there local differences?[J]. J Gastroenterol Hepatol, 2007,22:78893. https://doi.org/ 10.1111/j.1440-1746.2007.05042.x.

14. Ahmed A, Wong RJ, Harrison SA. Nonalcoholic Fatty Liver Disease Review: Diagnosis, Treatment, and Outcomes[J]. Clin Gastroenterol Hepatol, 2015,13:2062-70. https://doi.org/ https://doi.org/10.1016/j.cgh.2015.07.029.

15. Younossi ZM, Otgonsuren M, Henry L, et al. Association of nonalcoholic fatty liver disease (NAFLD) with hepatocellular carcinoma (HCC) in the United States from 2004 to 2009[J]. Hepatology, 2015,62:1723-30. https://doi.org/ 10.1002/hep.28123.

16. Pais R, Lebray P, Rousseau G, et al. Nonalcoholic Fatty Liver Disease Increases the Risk of Hepatocellular Carcinoma in Patients With Alcohol-Associated Cirrhosis Awaiting Liver Transplants[J]. Clin Gastroenterol Hepatol, 2015,13:992-9. https://doi.org/ https://doi.org/10.1016/j.cgh.2014.10.011.

17. Liu H, Lu HY. Nonalcoholic fatty liver disease and cardiovascular disease[J]. World J Gastroenterol. 2014;20:8407-15. https://doi.org/10.3748/wjg.v20.i26.8407.

18. Musso G, Gambino R, Tabibian JH, et al. Association of non-alcoholic fatty liver disease with chronic kidney disease: a systematic review and meta-analysis[J]. PLoS Med. 2014;11:e1001680. https://doi.org/10.1371/journal.pmed.1001680.

19. Stefan N, Fritsche A, Schick F, et al. Phenotypes of prediabetes and stratification of cardiometabolic risk[J]. Lancet Diabetes Endocrinol. 2016;4:789-98. https://doi.org/10.1016/S2213-8587(16)000826.

20. Milic S, Lulic D, Stimac D. Non-alcoholic fatty liver disease and obesity: biochemical, metabolic and clinical presentations[J]. World J Gastroenterol, 2014,20:9330-7. https://doi.org/ 10.3748/wjg.v20.i28.9330.

21. Liu CJ. Prevalence and risk factors for non-alcoholic fatty liver disease in Asian people who are not obese[J]. J Gastroenterol Hepatol, 2012,27:1555-60. https://doi.org/ 10.1111/j.14401746.2012.07222.x.

22. Katsiki N, Mikhailidis DP, Mantzoros CS. Non-alcoholic fatty liver disease and dyslipidemia: An update[J]. Metabolism, 2016,65:1109-23. https://doi.org/ https://doi.org/10.1016/j.metabol.2016.05.003.

23. Targher G, Bertolini L, Rodella S, et al. NASH predicts plasma inflammatory biomarkers independently of visceral fat in men[J]. Obesity (Silver Spring), 2008,16:1394-9. https://doi.org/ 
10.1038/oby.2008.64.

24. Imajo K, Hyogo H, Yoneda M, et al. LDL-migration index (LDL-MI), an indicator of small dense lowdensity lipoprotein (sdLDL), is higher in non-alcoholic steatohepatitis than in non-alcoholic fatty liver: a multicenter cross-sectional study[J]. PloS one, 2014,9:e115403. https://doi.org/ 10.1371/journal.pone.0115403.

25. Norris AL, Steinberger J, Steffen LM, et al. Circulating oxidized LDL and inflammation in extreme pediatric obesity[J]. Obesity (Silver Spring), 2011,19:1415-9. https://doi.org/ 10.1038/oby.2011.21.

26. Sinn DH, Gwak G, Park HN, et al. Ultrasonographically detected non-alcoholic fatty liver disease is an independent predictor for identifying patients with insulin resistance in non-obese, non-diabetic middle-aged Asian adults[J]. Am J Gastroenterol, 2012,107:561-7. https://doi.org/ 10.1038/ajg.2011.400.

27. Lewis GF, Murdoch S, Uffelman K, et al. Hepatic lipase mRNA, protein, and plasma enzyme activity is increased in the insulin-resistant, fructose-fed Syrian golden hamster and is partially normalized by the insulin sensitizer rosiglitazone[J]. Diabetes, 2004,53:2893-900. https://doi.org/ 10.2337/diabetes.53.11.2893.

28. Speliotes EK, Massaro JM, Hoffmann U, et al. Fatty liver is associated with dyslipidemia and dysglycemia independent of visceral fat: the Framingham Heart Study[J]. Hepatology, 2010,51:1979-87. https://doi.org/ 10.1002/hep.23593.

29. Go GW. Low-Density Lipoprotein Receptor-Related Protein 6 (LRP6) Is a Novel Nutritional Therapeutic Target for Hyperlipidemia, Non-Alcoholic Fatty Liver Disease, and Atherosclerosis[J]. Nutrients, 2015,7:4453-64. https://doi.org/ 10.3390/nu7064453.

30. Go G, Srivastava R, Hernandez-Ono A, et al. The Combined Hyperlipidemia Caused by Impaired WntLRP6 Signaling Is Reversed by Wnt3a Rescue[J]. Cell Metab, 2014,19:209-20. https://doi.org/ https://doi.org/10.1016/j.cmet.2013.11.023.

31. Sun DQ, Wu SJ, Liu WY, et al. Association of low-density lipoprotein cholesterol within the normal range and NAFLD in the non-obese Chinese population: a cross-sectional and longitudinal study[J]. BMJ Open, 2016,6:e13781. https://doi.org/ 10.1136/bmjopen-2016-013781.

32. Sun D, Liu W, Wu S, et al. Increased levels of low-density lipoprotein cholesterol within the normal range as a risk factor for nonalcoholic fatty liver disease[J]. Oncotarget, 2016,7:5728. https://doi.org/ 10.18632/oncotarget.6799.

33. Vandenbroucke JP, von Elm E, Altman DG, et al. Strengthening the Reporting of Observational Studies in Epidemiology (STROBE): Explanation and elaboration[J]. Int J Surg, 2014,12:1500-24. https://doi.org/ https://doi.org/10.1016/j.ijsu.2014.07.014.

34. Kernan WN, Viscoli CM, Brass LM, et al. Phenylpropanolamine and the risk of hemorrhagic stroke[J]. N Engl J Med, 2000,343:1826-32. https://doi.org/ 10.1056/NEJM200012213432501.

\section{Figures}




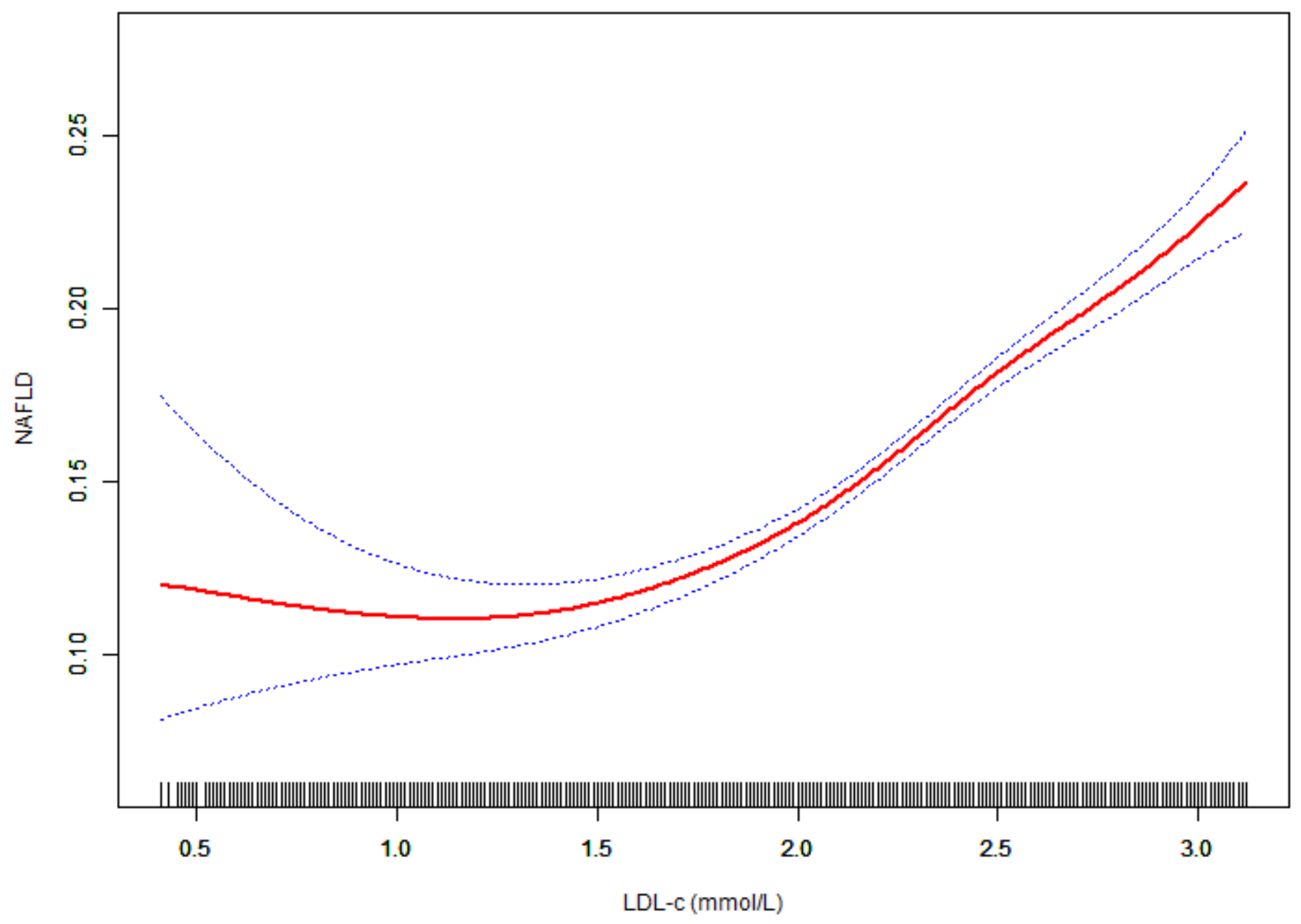

Figure 1

The non-linear relationship between LDL-C and NAFLD. A non-linear relationship between them was detected after adjusting for AGE, SEX, BMI, ALT, AST, GGT, BUN, Cr, TG, FPG, TC, UA, HDL-C, GLB and ALB

\section{Supplementary Files}

This is a list of supplementary files associated with this preprint. Click to download.

- SupplementaryMaterial.docx

- SupplementaryMaterial.docx 